\title{
Neonatal mouse ileum: functional properties and protein composition of the contractile machinery
}

\author{
Frank Eifinger 1,2, Lubomir T. Lubomirov², Elena Dercks ${ }^{1,2}$, Borislav Genchev³, Bernhard Roth', Wolfram F. Neiss ${ }^{3}$, Gabriele Pfitzer ${ }^{2}$
} and Mechthild M. Schroeter ${ }^{2}$

BACKGROUND: Immature motility of the ileum may contribute to life-threatening diseases. Little is known about the normal biomechanics of the neonatal ileum in relation to the protein composition of its contractile machinery.

METHODS: We analyzed the tissue architecture, the biomechanics in intact and $\beta$-escin-permeabilized preparations, and the protein composition in neonatal (PO) and adult murine ileum.

RESULTS: Muscle thickness of the P0 ileum was $-50 \%$ of the adult ileum and passive compliance was higher. Carbachol- and $\mathrm{KCl}$-elicited contractions were tonic rather than phasic as in the adult. $\mathrm{Ca}^{2+}$ sensitivity was higher and relaxation rate was slower in $\beta$-escin-permeabilized PO compared with adult ileum. The expression level of $\beta$-actin relative to a-actin was higher, and those of total actin, myosin, myosin light chain kinase, the catalytic subunit of myosin phosphatase and telokin were lower compared with the adult. The expression level of MYPT1 was similar, but P0 ileum expressed only the M133; the adult ileum also expressed the M130 isoform.

CONCLUSION: The mechanical features and protein composition of the PO ileum are similar to those of adult tonic smooth muscles. We propose that this is highly adaptive during fetal life allowing the small intestine to act predominantly as a container.

D uring development from fetal to newborn and adult life, the gastrointestinal tract (GIT) gradually establishes its mature structure and function. Anatomic features of the digestive tract are present already in human fetal life by $8 \mathrm{wk}$ gestational age (1). However, gastrointestinal motility of preterm individuals is reduced (2). Obviously, this poses severe problems regarding oral food intake which are aggravated by the fact that the immaturity of the GIT, singly or in concert with other perinatal risk factors, may contribute to complications such as spontaneous intestinal perforation, not uncommon in extremely low-birth-weight infants (3). The underlying cause of spontaneous intestinal perforation, one of the leading risk factors of death in neonatal medicine, is still poorly understood. Conceivably an immature intestinal motility is an important determinant leading to stasis of the intestinal contents and overgrowth of infectious enteritis pathogens (4). The ensuing inflammatory response leads to a viscous cycle by not only further impairing of propulsive gut movements but also derogating vascular smooth muscle (SM) function, leading to reduced blood supply and finally to necrosis.

Intestinal motility is driven by the concerted action of enteric neurons, pacemaker, and SM cells (2). Although several studies addressed different aspects of the fetal and postnatal maturation of innervation, electrical activity, and global contractile activity of the GIT (5-7), only few studies addressed in some depth the properties of the downstream effector, the SM. Investigations from avian (8) and mammalian stomach (911 ), and guinea pig gallbladder (12) as well as from non-GIT organs like urinary bladder $(13,14)$, revealed major differences between neonatal and adult tissues as regards electro- and pharmacomechanical coupling $(14,15)$, protein composition, and functional properties of the contractile apparatus (14-17). Interestingly, despite the widespread use of transgenic mouse models to investigate the contribution of individual proteins to the regulation of contraction (18-20), we are not aware of a systematic evaluation of biomechanics of the neonatal ileum coincident with its tissue architecture and protein composition of the contractile machinery.

In this study, we report major differences in the length-tension relation of murine neonatal (P0) ileum and a shift from adult phasic to tonic contractions. These functional differences can be ascribed to differences in the tissue architecture and protein composition of the contractile machinery, which is similar to that found in adult tonic SM (e.g., 8;21;22), whereas the higher expression level of $\beta$-actin and nonmuscle myosin heavy chain (NM-MHC) reflects the immaturity of the neonatal ileum. Because the P0 mouse is less mature compared with the newborn human, we propose that the properties reported here may reflect the situation of prematurely born infants.

\section{RESULTS}

Histology and Expression of Contractile Proteins

Like the adult ileum, the neonatal murine ileum consists of four main layers (Figure 1a-d): (i) the outermost extremely 
a

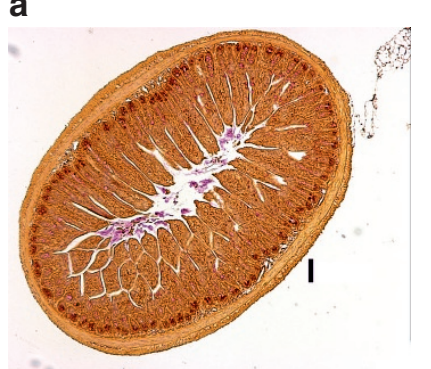

b

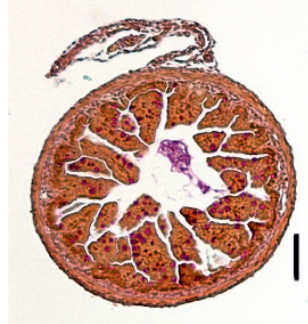

C

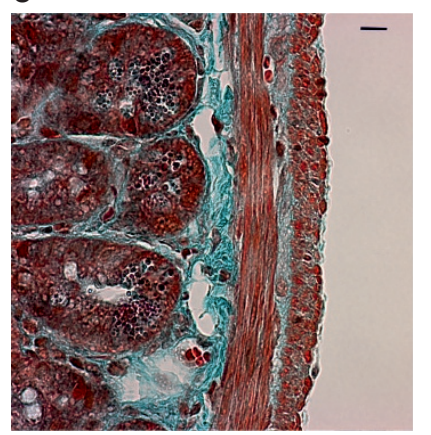

d

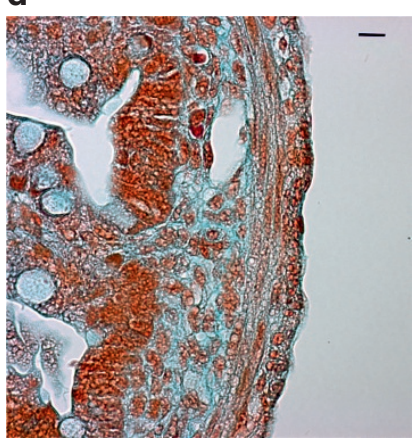

e

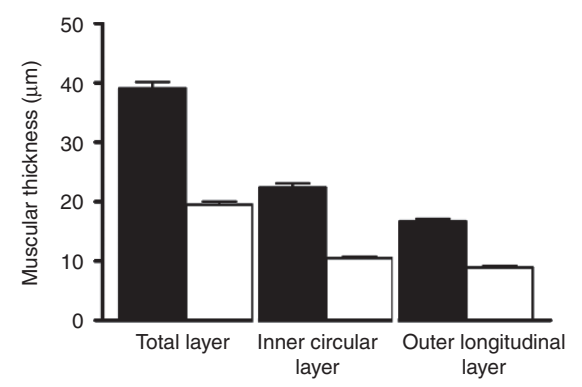

Figure 1. Cross-sections of adult $(\mathbf{a}, \mathbf{c})$ and $P 0(\mathbf{b}, \mathbf{d})$ ileum and morphometry of the tunica muscularis (e). Tri-PAS (a,b, scale bar $=100 \mu \mathrm{m})$, MassonGoldner staining (c,d, scale bar $=10 \mu \mathrm{m})$, (e) bars (black, adult; white, P0) represent mean \pm SEM, $n=10$. Tri-PAS, periodic acid-Schiff-orange G.

thin tunica serosa (1-2 cell layers), (ii) the tunica muscularis, (iii) the very thin tunica or tela submucosa, and (iv) the innermost tunica mucosa with long villi and shorter crypts that in the adult gut contained numerous Paneth cells loaded with an abundance of periodic acid-Schiff-orange G-positive and hematoxylin-stainable secretory granules (Figure 1a,c). The lamina muscularis mucosa-highly characteristic of the human tunica mucosa-was almost missing in neonate and very thin in the adult ileum. The SM cells in the neonatal ileum appeared less densely packed and more interspersed with connective tissue. Although the boundaries were less marked in P0 ileum, the outer longitudinal and inner circular muscle layer could be clearly identified (Figure 2a). The thickness of both muscle layers was reduced by $-50 \%$ in $\mathrm{P} 0$ compared with adult ileum (Figure 1e). The total protein content per wet weight of the tunica muscularis from neonatal and adult ileum was similar in both age groups (Figure $2 \mathbf{b}$ ). The actin and myosin content was significantly lower, and the molar ratio of actin to myosin was higher than in the adult (Figure $2 c-e$ ).

\section{Length-Tension Relationship of Neonatal and Adult Ileal SM}

Compared with the adult ileum, the passive length-tension relation of P0 ileum was strikingly flat (Figure 3a,b) and $\mathrm{KCl}$ induced force increased only up to $1.2 \mathrm{~L}_{0}$ remaining constant thereafter (Figure 3b), whereas in the adult ileum, active force steeply increased up to $1.9 \mathrm{~L}_{0}$ (Figure 3a, c.f.) (23). At all lengths, $\mathrm{KCl}$ induced a rapid increase in force (peak-1) followed by a decline in force to near resting levels in the adult ileum (Figure 3c), whereas in the P0 ileum (Figure 3d), the rise in force was slower and the subsequent relaxation was less $(-50 \%$ compared with $-90 \%$ in the adult). The tonic component of the contraction was maintained for at least $5 \mathrm{~min}$. At optimal length, absolute peak force was lower in the P0 $(0.46 \pm 0.08$ $\mathrm{mN}$ at $\left.1.2 \mathrm{~L}_{0}\right)$ compared with adult ileum $(1.82 \pm 0.27 \mathrm{mN}$ at $1.9 \mathrm{~L}_{0}$ ) as was specific force (Figure $3 \mathrm{e}$ ). Raising the temperature from $21^{\circ} \mathrm{C}$ to $37^{\circ} \mathrm{C}$ had no significant effect on the pattern of $\mathrm{KCl}$-induced contractions but increased the amplitude and frequency of the spontaneous rhythmic activity in both age groups (Figure 4). Of note, the rhythmic activity of neonatal, but not of the adult ileum, was regular at both temperatures.

\section{Carbachol (CCh)-Induced Contractions}

The pattern of the CCh-induced contractions depended on the $\mathrm{CCh}$ concentration in both age groups (Figure 5a-d). Low concentrations of CCh $(<0.3 \mu \mathrm{mol} / \mathrm{l})$ induced a transient increase in the amplitude of the rhythmic contractions. With increasing concentrations, a tonic component with superimposed rhythmic contractions became apparent, the threshold concentration for the appearance of the tonic component being lower in the neonate. Finally, the rhythmic activity subsided and the initial fast rise in force (peak-1) was followed by a partial relaxation in the adult ileum and by a slower rise to a sustained force in P0 ileum (c.f. also Supplementary Figure S1 online). The $\mathrm{pEC}_{50}$ values (Figure $5 \mathbf{e}, \mathbf{f}$ ) for peak- 1 were $6.7 \pm 0.2$ in neonatal and $7.4 \pm 0.1$ in adult ileum $(P<0.05)$ and for the tonic component evaluated $10 \mathrm{~min}$ after addition of CCh $6.1 \pm 0.1$ in the neonate and $5.2 \pm 0.2$ in the adult $(P<0.01)$. Different from adult ileum (24), the sustained force was relaxed by the ROK inhibitor Y-27632 with lower potency (Figure 6a-c). A protein kinase $\mathrm{C}$ inhibitor had a similar small effect on the tonic phase (c.f. Supplementary Figure S2 online).

\section{$\mathrm{Ca}^{2+}$ Sensitivity of Contraction in Permeabilized Ileum Is Higher in the Newborn Ileum}

Using membrane-permeabilized preparations, in which the myofilaments can be directly activated by $\mathrm{Ca}^{2+}$, we tested whether $\mathrm{Ca}^{2+}$ sensitivity and myosin light chain phosphatase (MLCP) activity in P0 ileum are similar to those of tonic adult SM (21) whereby the rate of relaxation and $\mathrm{MLC}_{20}$ dephosphorylation is taken as indirect estimate of MLCP activity. In P0 compared with adult ileum, the force- $\left[\mathrm{Ca}^{2+}\right]$ relation was shifted toward lower $\left[\mathrm{Ca}^{2+}\right]$, indicating a higher $\mathrm{Ca}^{2+}$ sensitivity (Figure 6d). Relaxation was slower than in adult ileum coincident with a slower time course of $\mathrm{MLC}_{20}$ dephosphorylation indicating a lower MLCP activity. The dependence of force 
a

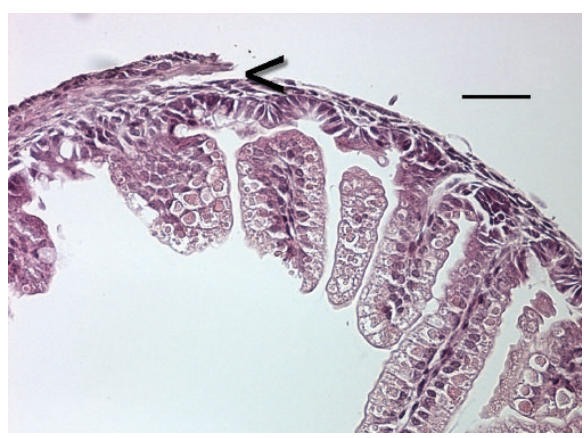

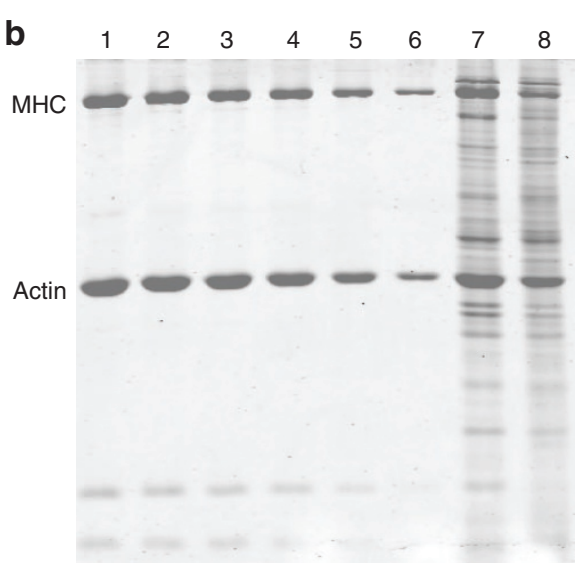

e

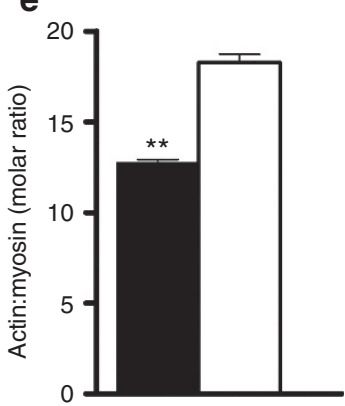

Figure 2. Determination of the actin and myosin content in neonatal and adult ileum. (a) HE stained cross-section of P0 ileum (scale bar $=50 \mu \mathrm{m}$ ) demonstrating that dissection separates the mucosa from the smooth muscle layer (arrowhead). (b) Coomassie blue-stained SDS-PAGE, lanes 1-6, decreasing concentrations of purified myosin and actin; 7, adult; and 8, P0 ileal smooth muscle. Panels c-e summarize results; black and white bars, adult and P0 ileum, respectively $(n=15) ;{ }^{*} P<0.05,{ }^{* *} P<0.01$.

decline on $\mathrm{MLC}_{20}$ dephosphorylation was similar in both age groups (Figure 6e,f).

\section{Protein Composition of the Contractile Machinery}

Figure $7 \mathbf{a}-\mathbf{f}$ shows the analysis of selected proteins of the contractile machinery of the mucosa-free muscular layer. As an index of maturity, the expression levels of $\alpha$ - and $\beta$-actin, NM-MHC, and the fetal isoform of caldesmon (1-caldesmon) were determined. Expression of NM-MHC and $\beta$-actin was higher and that of $\alpha$-actin was lower than in the adult. Expression of 1-caldesmon was negligible in P0, whereas that of h-caldesmon, the isoform of mature SM, was similar in both groups $(P=0.49)$. Calponin was less expressed in the neonate. Expression of SMB, the myosin isoform of phasic adult SM (25), was lower in P0 ileum. Expression of the regulatory proteins involved in determining $\mathrm{Ca}^{2+}$ sensitivity (reviewed in Ref. (26)) revealed lower amounts of myosin light chain kinase (MLCK), the catalytic subunit of MLCP (PP1c $\delta)$, and telokin in P0 ileum, whereas expression of the regulatory subunit of MLCP, MYPT1, which targets PP1c $\delta$ to myosin, was similar in both groups. Mammalian SM expresses four, developmentally regulated MYPT-1 isoforms differing by a C-terminal leucine zipper domain and a central insert $(17,27)$. Isoforms containing the central insert with and without the leucine zipper domain have a lower electrophoretic mobility (28). In the adult tissue, the anti-MYPT1 $1_{\text {total }}$ antibody detected two immunoreactive bands most likely corresponding to the M133 and
M130 isoforms described in avian SM (8). In the neonatal tissue, only the upper band was detected.

\section{DISCUSSION}

The terminal ileum is a predilection site for spontaneous perforation in premature infants, which may be related to an immature gut motility (3). However, little is known about the biomechanics of the neonatal ileum. In this study, we present evidence of major functional differences of murine P0 compared with adult ileum. Its most prominent functional features are the high passive compliance and the switch of the contractile phenotype from the adult phasic toward a more tonic phenotype. This shift is reflected at the level of the contractile machinery by a high $\mathrm{Ca}^{2+}$ sensitivity and slow rate of relaxation coincident with a protein expression pattern previously described to be characteristic for adult tonic SMs.

\section{Force Length Relation and Myogenic Rhythmic Activity}

Compared with the adult ileum, the P0 ileum is highly compliant and active force-length relation is shallow. SM cells are embedded into an extracellular matrix, which together with the tissue architecture is a major determinant of the passive elastic properties (29). It is tempting to speculate that the immature architecture of the neonatal ileum (c.f. Figure 1) underlies this high compliance. Such a high compliance, also found in the neonatal stomach (9), may thus be characteristic of the neonatal GIT. 
a

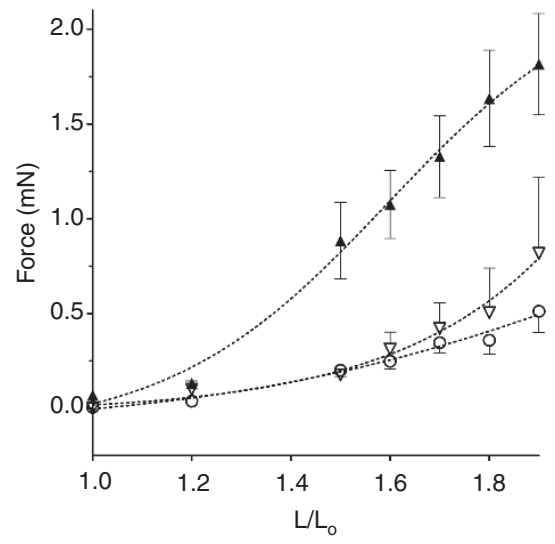

C

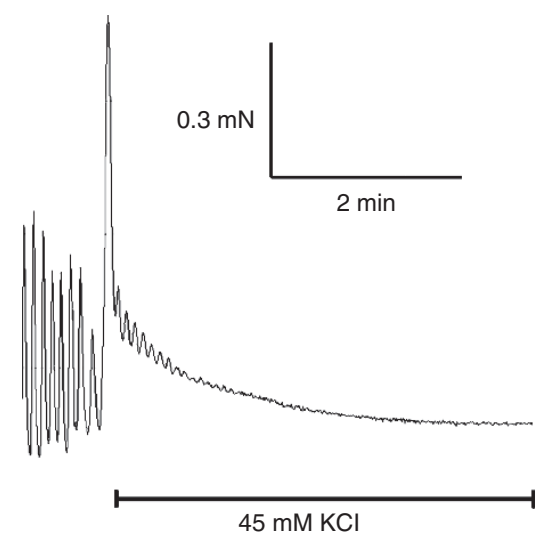

e

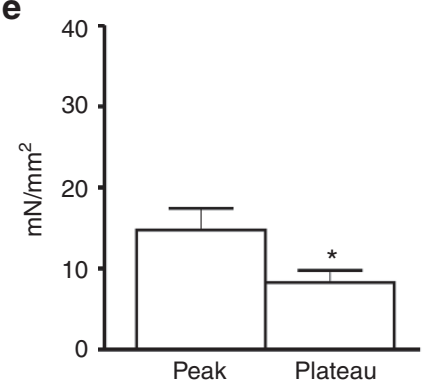

b

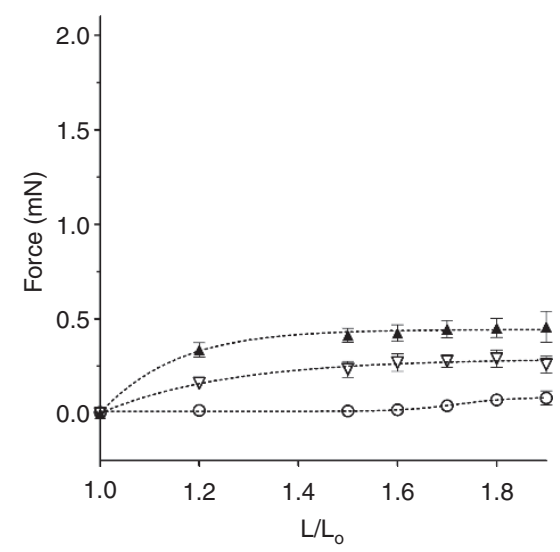

d
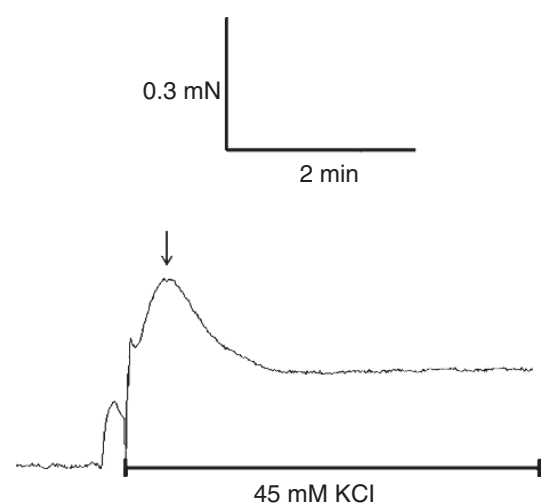

$45 \mathrm{mM} \mathrm{KCl}$

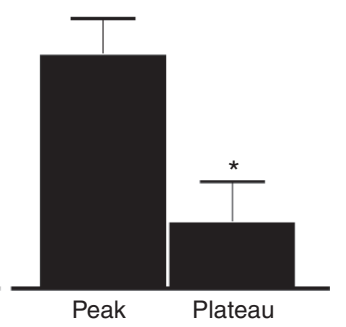

Figure 3. Active and passive length-tension relation of adult (a) and PO ileal smooth muscle (b). Passive force (circles), the initial peak (closed triangles), and plateau force (open triangles) determined $4 \mathrm{~min}$ after $45 \mathrm{mmol} / \mathrm{I} \mathrm{KCl}$ application were plotted vs. relative length $\left(\mathrm{L}_{0}=\right.$ slack length). Representative force responses to $\mathrm{KCl}(45 \mathrm{mmol} / \mathrm{l})$ of adult (c) and neonatal (d) ileum at, respectively, $1.6 \mathrm{~L}_{0}$ and $1.2 \mathrm{~L}_{0} ;$ arrow in $\mathbf{d}$ denotes peak; (e) $\mathrm{KCl}$-induced force per cross-sectional area (mN/mm², c.f. Supplementary Methods online) at $1.6 \mathrm{~L}_{0}$ (adult) and $1.2 \mathrm{~L}_{0}(\mathrm{PO}) ;$ white and black bars, $\mathrm{PO}$ and adult ileum, respectively $(n=10) ;{ }^{*} P<0.05$.

The developmental aspects of spontaneous rhythmic activity in vitro and of motor complexes in vivo have been studied in some detail before $(1,2,5-7,30)$. Slow waves generated by interstitial cells of Cajal can be detected in mice some $6 \mathrm{~h}$ after birth (5) having a similar frequency as the rhythmic contractions at $37^{\circ} \mathrm{C}$ in our study. Thus, slow waves may underly the spontaneous contractile activity of the P0 ileum. Their lower amplitude possibly reflects the reduced expression of MLCK (19). Although in the P0 mouse, neuronal activity does not contribute to rhythmic activity (reviewed in Ref. (2)), addition of CCh augmented the spontaneous activity, indicating that muscarinic receptors are functional.

\section{Excitation Contraction Coupling in Relation to Contractile Protein Expression}

Consistent with previous studies with different neonatal SMs $(8,9,11,12,14,31)$, we found that actin and myosin expression and specific active peak force elicited by $\mathrm{KCl}$ and carbachol at optimal length were lower compared with the adult. Specific force takes into account that the mechanical output depends on multiple factors, some of which are difficult to assess. These factors include mechanical coupling between myocytes and the extracellular matrix, and the number and width of SM cells acting in parallel $(9,10)$. At the subcellular level, force depends on the absolute content of the contractile proteins and the length of the sarcomere equivalents, longer 
a

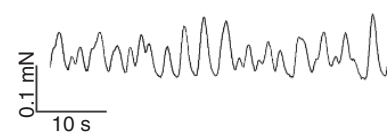

C

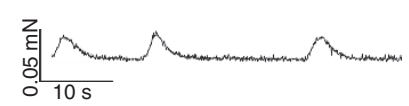

e

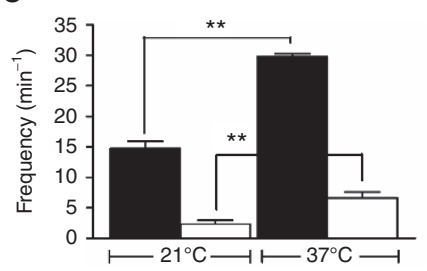

b

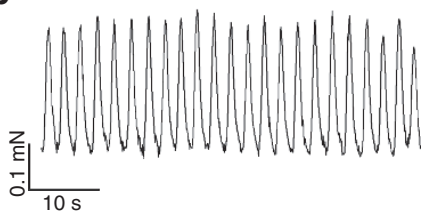

d

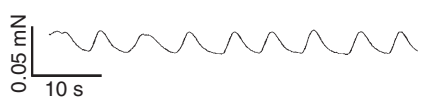

f

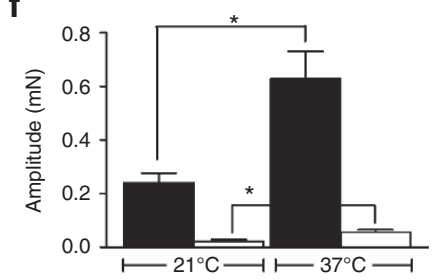

Figure 4. Spontaneous rhythmic activity. Original force recordings from adult $(\mathbf{a}, \mathbf{b})$ and PO (c,d) ileal smooth muscle at $21^{\circ} \mathrm{C}(\mathbf{a}, \mathbf{c})$ and $37^{\circ} \mathrm{C}(\mathbf{b}, \mathbf{d})$ at $1.4 \mathrm{~L}_{0}$ in both groups; (e,f) summary of results, black and white bars, adult and P0 ileum, respectively $(n=9-20) ;{ }^{*} P<0.05,{ }^{* *} P<0.01$.

ones allowing more myosin cross-bridges to act in parallel, and finally intracellular signaling mechanisms. As reported for other neonatal SM $(12,13)$, Rho/ROK- and protein kinase $\mathrm{C}$-induced $\mathrm{Ca}^{2+}$-sensitizing pathways seem to be of minor importance. In contrast, sustained force in adult ileum critically depends on activation of Rho/ROK (24). Confirming previous observations of maturational differences in excitation contraction coupling $(14,15,32)$ these results suggest that the tonic contraction of P0 ileum reflects intrinsic properties of the contractile machinery rather than G-protein-activated $\mathrm{Ca}^{2+}$-sensitizing pathways.

\section{Properties of the Contractile Machinery}

Adult SMs are classified into tonic, slow-contracting, and phasic, fast-contracting SMs depending on whether or not $\mathrm{KCl}$ elicits a sustained contraction (26). On the basis of this definition, the mature ileum is a phasic, whereas the P0 ileum is a tonic SM. Like in adult tonic SM, intrinsic $\mathrm{Ca}^{2+}$ sensitivity of the contractile machinery of P0 ileum is high and rates of $\mathrm{MLC}_{20}$ dephosphorylation and relaxation are slow (21). With the exception of caldesmon, the protein composition of the neonatal contractile machinery recapitulates that of adult, tonic SMs, e.g., lower expression of MLCK (21), expression of a MYPT-1 isoform likely corresponding to the M133 isoform described in tonic avian SM (8), and lower expression of the MLCP activator telokin (22). Similar findings have been reported for fetal chicken gizzard (8) and neonatal mouse bladder (14), both are phasic in the adult. It remains to be determined whether the M133 MYPT1 isoform contains the leucine zipper domain, which was proposed to be a marker of the developmental switch from the tonic to phasic contractile phenotype in rat portal vein (27). The lower expression

of calponin (33) in P0 ileum is likely not involved in the shift toward the tonic phenotype (34).

Detailed investigations in adult tonic and phasic SM revealed that their different mechanical properties are inherent to their myosin-II isozymes (35). Moreover, it was proposed that NM myosin-II being a "slow myosin" may be involved in sustained force $(20,25)$. The precise determination of the molar ratios of the different MHC isoforms in ileal SM was beyond the scope of this study, but our results (c.f. Figure 7) are consistent with a contribution of NM-MHC and reduced SMB expression (25) to the tonic phenotype-without excluding other scenarios such as a shift in MYPT1 isoforms (8). Interestingly, contractions of MYPT1-deficient murine adult ileum are tonic (18). Here, one has to remember that MYPT1 expression in P0 and adult ileum is similar. Thus, our results indicate that under physiological conditions, the contractile phenotype is determined by the specific blend of the protein composition of the contractile machinery rather than by a single protein.

$\mathrm{Ca}^{2+}$ sensitivity of force is predominantly determined by the MLCK/MLCP activity ratio (26). The high intrinsic $\mathrm{Ca}^{2+}$ sensitivity, a hallmark of tonic SM, is likely due to the lower MLCP activity (21). In chicken gizzard, expression of the M133 isoform was associated with a slower rate of relaxation $(8,17)$ and $\mathrm{MLC}_{20}$ dephosphorylation (8). This and the lower expression of PP1c $\delta$ and telokin (c.f. Figure7) predict a lower activity of MLCP, which is reflected in the slower dephosphorylation of $\mathrm{MLC}_{20}$ after removal of $\mathrm{Ca}^{2+}$ and consequent inactivation of MLCK. We note, that the lower MLCP activity is not due to G-protein-induced inhibition $(24,26)$ because these pathways are not activated under our experimental conditions. Because P0 and adult ileum exhibit a similar dependence of force decline on $\mathrm{MLC}_{20}$ dephosphorylation, relaxation may largely depend on MLCP activity without excluding the possibility that it is further rate limited by myosins with a slow rate of cross-bridge detachment such as NM myosin II $(20,25)$ or slow SM myosin II isozymes, the latter being expressed in adult tonic SM (35) and possibly also in P0 ileum. The lower expression level of MLCK does not necessarily imply a lower MLCK/MLCP activity ratio as the specific activity of MLCK may be upregulated like in fetal carotid arteries (36) and a high $\mathrm{Ca}^{2+}$ sensitivity may be supported by $\mathrm{Ca}^{2+}$ independent MLC kinases (37). Their involvement in the P0 ileum remains to be determined.

The thin filament-linked protein caldesmon, the expression of which is higher in adult phasic compared with tonic SM, modulates relaxation kinetics (38). The fact that expression of caldesmon is not reduced in the $\mathrm{P} 0$ ileum raises the interesting possibility that caldesmon underlies the faster relaxation rate of the P0 ileum compared with purely tonic fetal gizzard (8) and adult femoral artery (21).

To summarize, our results support the notion of Ogut and Brozovich (8) that the tonic phenotype is the default for SM early in development. However, despite having tonic properties at the contractile machinery level, membrane-intact neonatal bladder responds to stimulation with CCh predominantly with an increased amplitude of the rhythmic contractions and only 


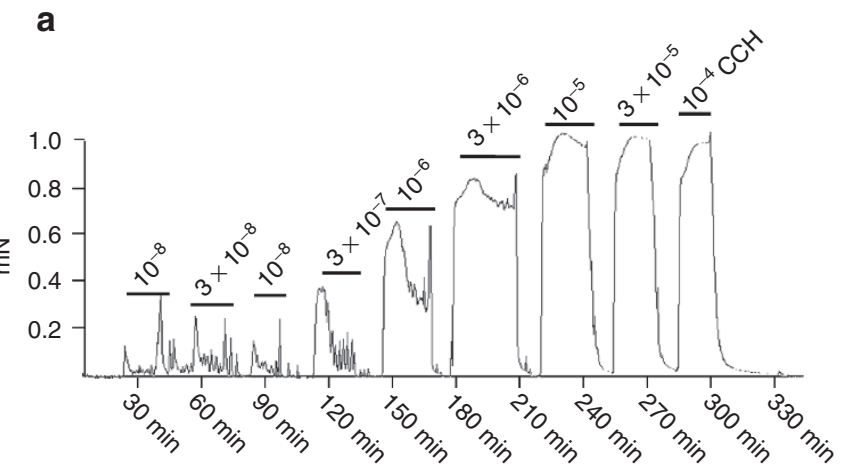

b
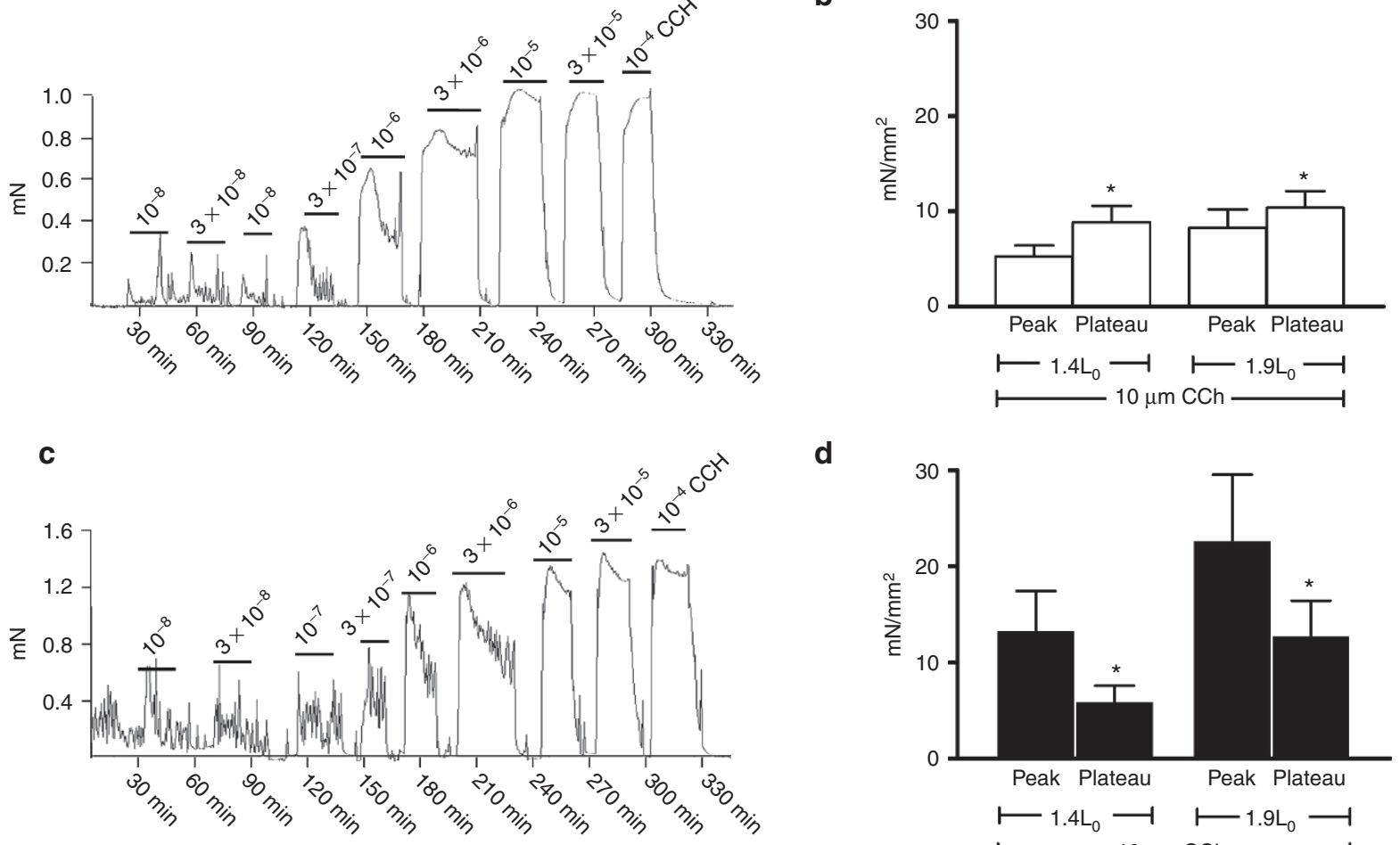

d

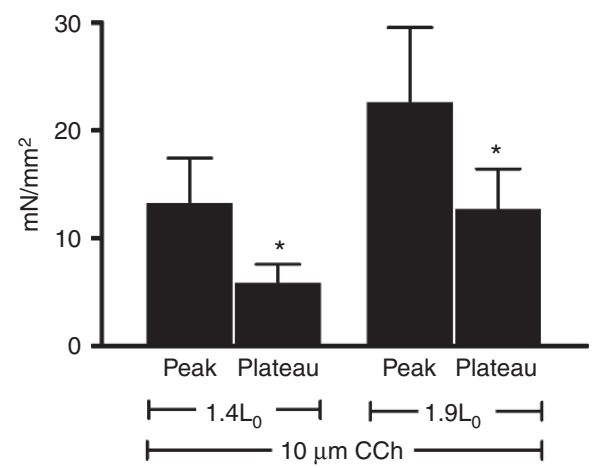

e

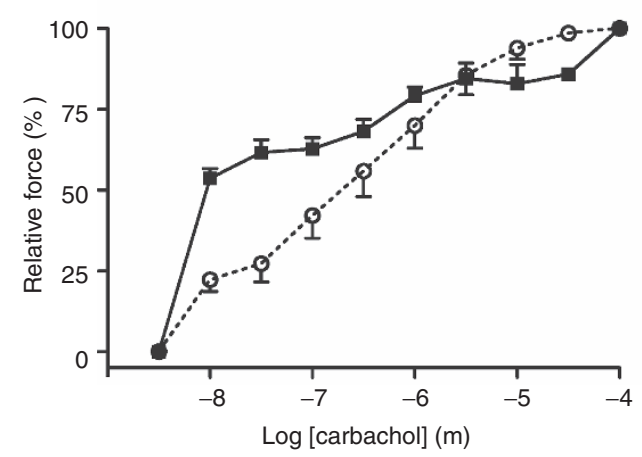

f

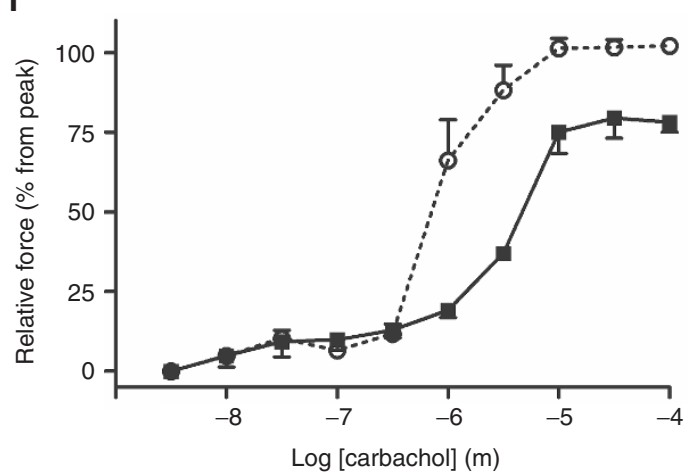

Figure 5. Noncumulative dose-response curves to carbachol (CCh). (a,c) Representative force tracings of PO (a) and adult (c) ileum prestretched to, respectively, $1.2 \mathrm{~L}_{0}$ and $1.4 \mathrm{~L}_{0^{\prime}}(\mathbf{b}, \mathbf{d})$ specific force at two lengths (black bars, adult; white bars, $\left.\mathrm{P} 0\right),(n=3-5) ;{ }^{*} P<0.05$. (e,f) Summary of dose-response curves, (e) peak-1 expressed relative to $10^{-4} \mathrm{~mol} / \mathrm{l} \mathrm{CCh}$, (f) plateau force relative to peak-1 for each CCh concentration; closed squares, adult; open circles, P0, $n=10$.

a modest rise in tonic force (13). Thus, organ-specific membrane properties seem to determine whether the tonic feature of the contractile machinery emerges at the cellular and tissue level.

\section{Clinical Relevance}

We propose that these functional differences are highly adaptive. The low compliance of the bladder, which has been attributed to stretch-induced $\mathrm{Ca}^{2+}$ influx (39), and its strong rhythmic contractions in response to CCh may oppose filling and support active fetal urinary bladder emptying. The bladder is emptied in utero up to 30 times per day in humans (40), which is of vital importance being one of the major sources of amnion fluid. In contrast, the high passive compliance together with the fivefold to 10-fold lower frequency and amplitude of the spontaneous rhythmic activity allows the GIT to function as a container which stores the accumulating content with little increase in wall stress and only slowly transports it aborally during fetal life (1). Indeed, any kind of propulsive motility must be avoided to prevent intrauterine excretion of meconium into amniotic fluid leading to fatal outcome of the unborn. Unfortunately, persistence of these highly adaptive properties in the extremely low-birth-weight may be counterproductive because it may contribute to the common problem of delayed stool passage (2) or stasis of the intestinal contents favoring bacterial overgrowth, a putative risk factor of spontaneous intestinal perforation. A limitation of our study is that we only investigated the mechanical properties of murine P0 
a

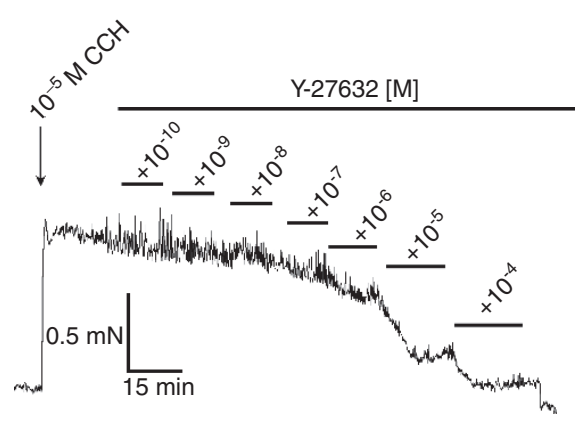

C
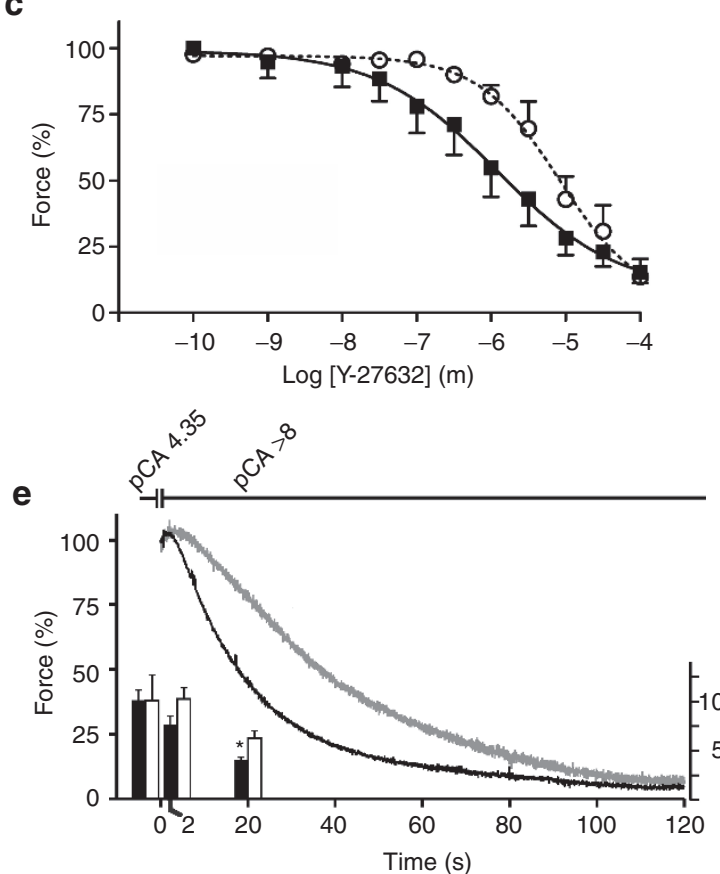

b
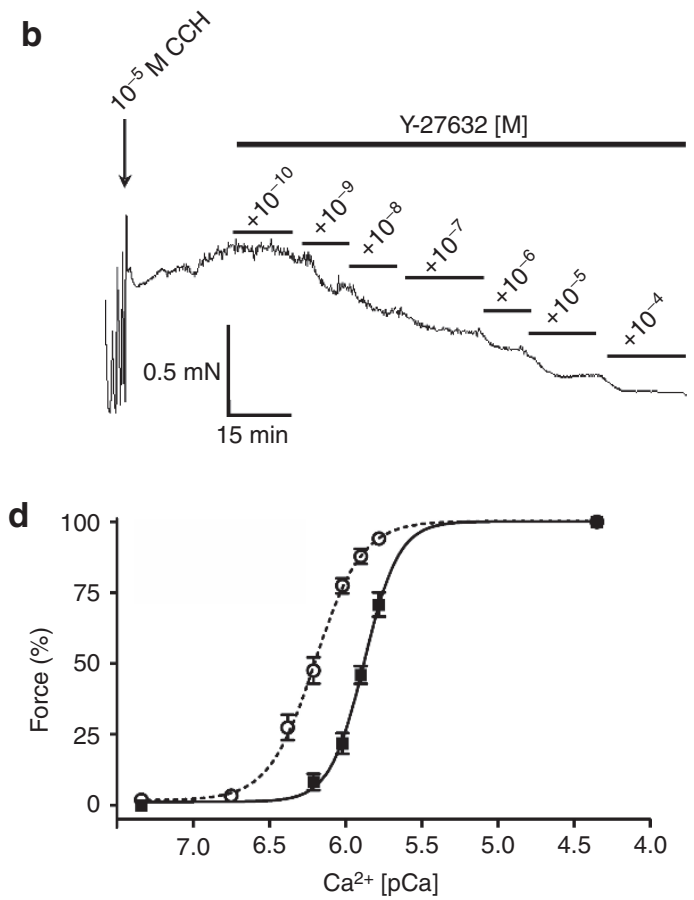

f

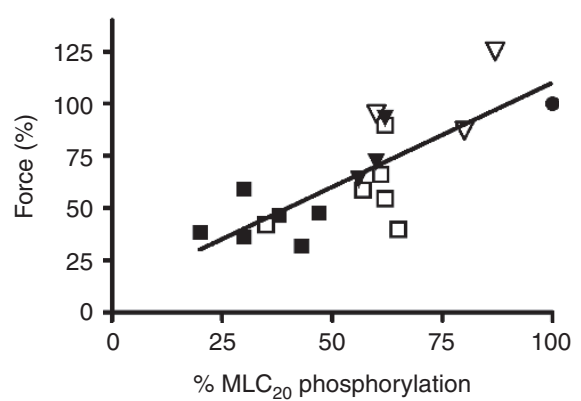

Figure 6. The Rho-kinase inhibitor Y-27632 induced a dose-dependent relaxation after precontraction with $10^{-5} \mathrm{~mol} / \mathrm{l} \mathrm{CCh}$. Original force tracings (a) P0 at $1.2 \mathrm{~L}_{0}$ and (b) adults at $1.4 \mathrm{~L}_{\rho^{\prime}}$ (c) summarizes results ( $\mathrm{plC}_{50}$-values: $5.15 \pm 0.19 \mathrm{P0}, 6.17 \pm 0.3$ adult, $P<0.05$ ); (d) force-pCa relations in $\beta$-escinpermeabilized PO (open circles) and adult ileal SM (closed squares); $\mathrm{PCa}_{50}$ : P0 6.29 \pm 0.25 , adult 5.86 $\pm 0.1, P<0.05$ ( $n=10$ ). (e) Time course of relaxation (black line, adult; grey line, P0) and $\mathrm{MLC}_{20}$ dephosphorylation (black bars, adult; white bars, $\mathrm{PO}, n=3-6$; ${ }^{*} P<0.05$; c.f. also Supplementary Figure S3 online) of $\beta$-escin-permeabilized ileal SM strips. Relaxation was induced by switching from $\mathrm{pCa} 4.35$ to $\mathrm{pCa}>8$ solution; half time of relaxation: $\mathrm{T}_{1 / 2 \text { adult }}$ $20.9 \pm 2.2 \mathrm{~s}, \mathrm{~T}_{1 / 2 n e o n a t e} 41.0 \pm 1.5 \mathrm{~s}, n=8, P<0.01$; (f) force-MLC 20 phosphorylation relation during relaxation; adult closed, $\mathrm{P} 0$ open symbols; triangles and squares, 2 and 20 s of relaxation; $100 \%=$ values at pCa 4.35 (closed circle).

ileum. It will be interesting to see how food intake affects maturation, how fast this occurs, and whether maturation is triggered by the mechanical load or by constituents of breast milk.

\section{METHODS}

\section{Tissue Dissection and Force Recording}

A detailed description is given in the Supplementary Methods online. All experiments complied with the European guidelines for the use of experimental animals and were approved by the local authorities (Bezirksregierung Nordrhein-Westfalen). All animals were sacrificed by cervical dislocation. The weights of newborn $(<24 \mathrm{~h}$ old, P0) and adult $(12 \mathrm{wk})$ male C57/BL6NCR mice were $1.72 \pm 0.1 \mathrm{~g}$ and $26.8 \pm 0.1 \mathrm{~g}$, respectively. The terminal ileum (length $2-6 \mathrm{~cm}$ ) was rapidly removed and flushed with physiological saline solution (PSS) containing $0.16 \mathrm{mmol} / \mathrm{CaCl}_{2}$ (low Ca-PSS). Crosssections of the P0 and adult ileum were stained with hematoxylin and eosin, periodic acid-Schiff-Orange G (Tri-PAS), and Masson's trichrome. For isometric force recording, mucosa-free muscle strips (width $1.6 \mathrm{~mm}$, length $8-10 \mathrm{~mm}$ ) were mounted in the direction of the longitudinal muscle layer in an organ bath. After determining slack length $\left(\mathrm{L}_{0}\right)$ in low Ca-PSS, the strips were allowed to equilibrate in high Ca-PSS containing $1.6 \mathrm{mmol} / \mathrm{l} \mathrm{CaCl}_{2}$ for $30-40 \mathrm{~min}$. Unless otherwise stated, experiments were performed at room temperature $\left(21-23{ }^{\circ} \mathrm{C}\right)$. Carbachol dose-response curves were obtained in a noncumulative manner at $1.4 \mathrm{~L}$ in adult and $1.2 \mathrm{~L}$ in neonatal strips. $\mathrm{Ca}^{2+}$ sensitivity relaxation kinetics and dephosphorylation of $\mathrm{MLC}_{20}$ at Ser19 during relaxation were determined in $\beta$-escin membranepermeabilized ileal strips.

\section{Protein Composition}

Protein lysates of the muscular layer were separated by sodium dodecyl sulfate-polyacrylamide gel electrophoresis. The actin and myosin contents were determined from Coomassie G250-stained gels using purified proteins as standards. The relative contents of SM $\alpha$ - and $\beta$-actin, NM-MHC, SM-myosin heavy chain B (SMB), MYPT$1, \mathrm{PP} 1 \mathrm{c} \delta$, MLCK, calponin, caldesmon, telokin, and of $\mathrm{MLC}_{20}$ phosphorylation were determined with western blot analysis. 
a

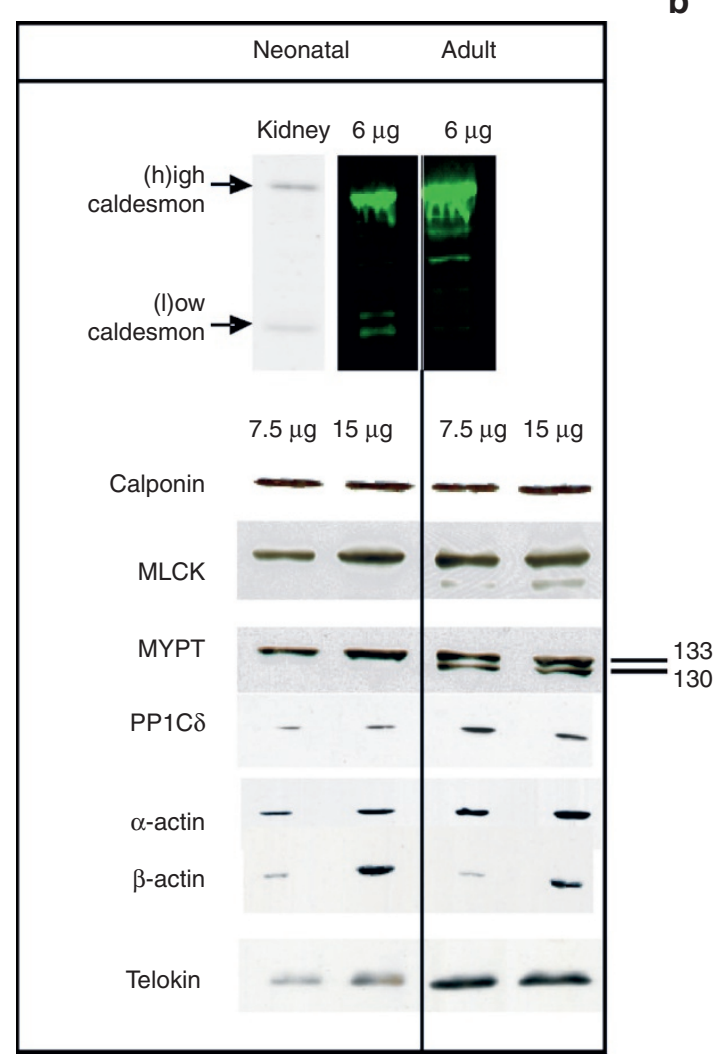

e

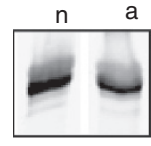

Total

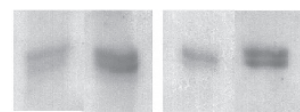

b

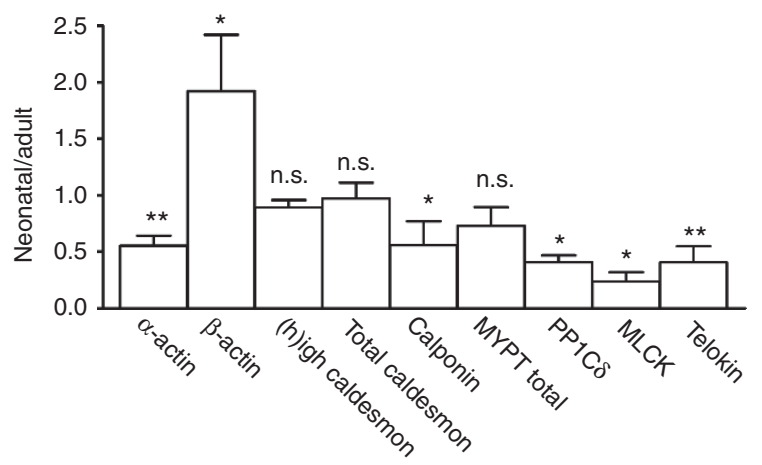

C

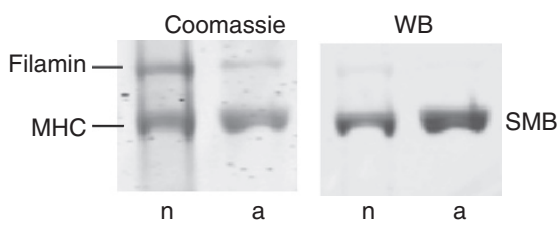

d

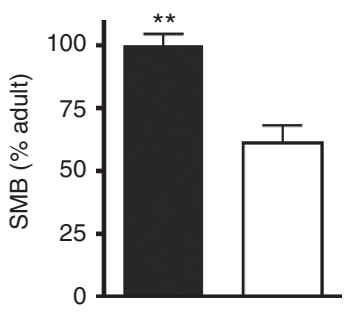

f

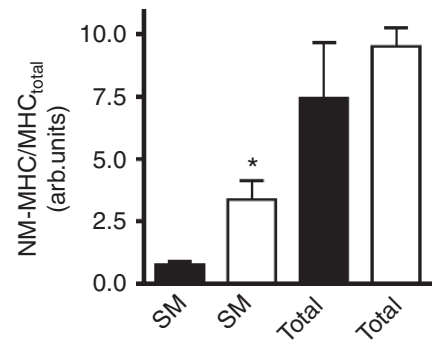

Figure 7. Expression of proteins of the contractile machinery. (a) Representative western blots: protein loading was 7.5 and $15 \mu \mathrm{g}$ in both groups; kidney tissue was used to identify $\mathrm{h}$ - and l-caldesmon. Adult and neonatal samples were run on the same SDS-PAGE, (b) expression of the immunoreactive signal of P0 ileum relative to that of adult ileum $(n=6)$; n.s., not significant; ${ }^{*} P<0.05,{ }^{*} P<0.01$. (c) Representative western blot with anti-SMB antibodies (right), corresponding Coomassie-stained gel (left), (d) summary of SMB expression of P0 ileum (white bar) relative to adult (black bar), $n=6,{ }^{* *} P<0.01$. (e) NM-MHC expression: western blot and Ponceau-S stained blot (corresponding lanes were cut out from the gel shown in Supplementary Figure $\mathbf{S 3}$ online). (f) Summary of NM-MHC expression in adult (black bars) and neonatal P0 (white bars) ileal SM and total ileum, i.e., SM + mucosa, $n=3,{ }^{*} P<0.05$; (for mucosa alone c.f. Supplementary Figure $\mathbf{S 4}$ online).

\section{Statistics}

All data are presented as mean \pm SEM with $n$ equaling the number of animals. The data were analyzed by two-way ANOVA, followed by Bonferroni post-test or Student's $t$-test when appropriate; $P<0.05$ was considered to indicate significant differences between groups.

\section{SUPPLEMENTARY MATERIAL}

Supplementary material is linked to the online version of the paper at http://www.nature.com/pr

\section{ACKNOWLEDGMENTS}

We thank AV Somlyo, D Hartshorne, I Morano, and TJ Eddinger for the generous gift of antibodies, and Doris Metzler and Ilona Rohrmann for their excellent technical assistance.

\section{STATEMENT OF FINANCIAL SUPPORT}

This work was supported by the KölnFortune program of the Medical Faculty of the University of Cologne (grant no. 61/2008 and 94/2010 to F.E.), and the Deutsche Forschungsgemeinschaft, Germany, to G.P. (SFB612-B12). The funding sources had no influence on the study design; in the data collection, analysis, and interpretation; in the writing of the report; and in the decision to submit the paper for publication.

Disclosure: The authors declare no conflict of interest.

\section{REFERENCES}

1. Anderson RB, Enomoto H, Bornstein JC, Young HM. The enteric nervous system is not essential for the propulsion of gut contents in fetal mice. Gut 2004;53:1546-7. 
2. Burns AJ, Roberts RR, Bornstein JC, Young HM. Development of the enteric nervous system and its role in intestinal motility during fetal and early postnatal stages. Semin Pediatr Surg 2009;18:196-205.

3. Gordon PV. Understanding intestinal vulnerability to perforation in the extremely low birth weight infant. Pediatr Res 2009;65:138-44.

4. Anand RJ, Leaphart CL, Mollen KP, Hackam DJ. The role of the intestinal barrier in the pathogenesis of necrotizing enterocolitis. Shock 2007;27:12433.

5. Liu LW, Thuneberg L, Huizinga JD. Development of pacemaker activity and interstitial cells of Cajal in the neonatal mouse small intestine. Dev Dyn 1998;213:271-82.

6. Roberts RR, Ellis M, Gwynne RM, et al. The first intestinal motility patterns in fetal mice are not mediated by neurons or interstitial cells of Cajal. J Physiol 2010;588(Pt 7):1153-69.

7. Sanders KM. Interstitial cells of Cajal at the clinical and scientific interface. J Physiol 2006;576(Pt 3):683-7.

8. Ogut O, Brozovich FV. Determinants of the contractile properties in the embryonic chicken gizzard and aorta. Am J Physiol Cell Physiol 2000;279:C1722-32.

9. Tomomasa T, Xie Y, Morikawa A, Kuroume T, Hyman PE. Postnatal changes in size and actomyosin content of rabbit gastric myocytes. Reprod Fertil Dev 1995;7:1305-10.

10. Tomomasa T, Yagi H, Kimura S, Snape WJ Jr, Hyman PE. Developmental changes in agonist-mediated gastric smooth muscle contraction in the rabbit. Pediatr Res 1989;26:458-61.

11. Ayas M, Bitar KN, Hillemeier C. Characterization of actin and myosin in the developing stomach. Pediatr Res 1995;37:202-6.

12. Camello-Almaraz C, Macias B, Gomez-Pinilla PJ, et al. Developmental changes in $\mathrm{Ca} 2+$ homeostasis and contractility in gallbladder smooth muscle. Am J Physiol Cell Physiol 2009;296:C783-91.

13. Ekman M, Andersson KE, Arner A. Receptor-induced phasic activity of newborn mouse bladders is inhibited by protein kinase $\mathrm{C}$ and involves T-type Ca2+ channels. BJU Int 2009;104:690-7.

14. Ekman M, Fagher K, Wede M, Stakeberg K, Arner A. Decreased phosphatase activity, increased $\mathrm{Ca} 2+$ sensitivity, and myosin light chain phosphorylation in urinary bladder smooth muscle of newborn mice. J Gen Physiol 2005; 125:187-96

15. Sandoval RJ, Injeti ER, Gerthoffer WT, Pearce WJ. Postnatal maturation modulates relationships among cytosolic $\mathrm{Ca} 2+$, myosin light chain phosphorylation, and contractile tone in ovine cerebral arteries. Am J Physiol Heart Circ Physiol 2007;293:H2183-92.

16. Lin VK, Robertson JB, Lee IL, Zimmern PE, McConnell JD. Smooth muscle myosin heavy chains are developmentally regulated in the rabbit bladder. J Urol 2000;164:1376-80.

17. Dirksen WP, Vladic F, Fisher SA. A myosin phosphatase targeting subunit isoform transition defines a smooth muscle developmental phenotypic switch. Am J Physiol Cell Physiol 2000;278:C589-600.

18. He WQ, Qiao YN, Peng YJ, et al. Altered contractile phenotypes of intestinal smooth muscle in mice deficient in myosin phosphatase target subunit 1. Gastroenterology 2013;144:1456-65, 1465.e1-5.

19. He WQ, Peng YJ, Zhang WC, et al. Myosin light chain kinase is central to smooth muscle contraction and required for gastrointestinal motility in mice. Gastroenterology 2008;135:610-20.

20. Löfgren M, Ekblad E, Morano I, Arner A. Nonmuscle Myosin motor of smooth muscle. J Gen Physiol 2003;121:301-10.
21. Gong MC, Cohen P, Kitazawa T, et al. Myosin light chain phosphatase activities and the effects of phosphatase inhibitors in tonic and phasic smooth muscle. J Biol Chem 1992;267:14662-8.

22. Choudhury N, Khromov AS, Somlyo AP, Somlyo AV. Telokin mediates $\mathrm{Ca} 2+$-desensitization through activation of myosin phosphatase in phasic and tonic smooth muscle. J Muscle Res Cell Motil 2004;25:657-65.

23. Gordon AR, Siegman MJ. Mechanical properties of smooth muscle. I. Length-tension and force-velocity relations. Am J Physiol 1971;221:12439.

24. Puetz S, Lubomirov LT, Pfitzer G. Regulation of smooth muscle contraction by small GTPases. Physiology (Bethesda) 2009;24:342-56.

25. Eddinger TJ, Meer DP. Myosin II isoforms in smooth muscle: heterogeneity and function. Am J Physiol Cell Physiol 2007;293:C493-508.

26. Somlyo AP, Somlyo AV. Ca2+ sensitivity of smooth muscle and nonmuscle myosin II: modulated by G proteins, kinases, and myosin phosphatase. Physiol Rev 2003;83:1325-58.

27. Payne MC, Zhang HY, Prosdocimo T, et al. Myosin phosphatase isoform switching in vascular smooth muscle development. J Mol Cell Cardiol 2006;40:274-82.

28. Huang QQ, Fisher SA, Brozovich FV. Unzipping the role of myosin light chain phosphatase in smooth muscle cell relaxation. J Biol Chem 2004;279:597-603.

29. Gabella G. Morphology of smooth muscle. In: Kao CY, Mary EC, eds. Cellular Aspects of Smooth Muscle Function. Cambridge, New York, Melbourne: Cambridge University Press, 1997:1-47.

30. Berseth CL. Gestational evolution of small intestine motility in preterm and term infants. J Pediatr 1989;115:646-51.

31. Seidel CL, Allen JC. Pharmacologic characteristics and actomyosin content of aorta from neonatal rats. Am J Physiol 1979;237:C81-6.

32. Zderic SA, Hypolite J, Duckett JW, Snyder HM 3rd, Wein AJ, Levin RM. Developmental aspects of bladder contractile function: sensitivity to extracellular calcium. Pharmacology 1991;43:61-8.

33. Kim HR, Appel S, Vetterkind S, Gangopadhyay SS, Morgan KG. Smooth muscle signalling pathways in health and disease. J Cell Mol Med 2008;12(6A):2165-80.

34. Szymanski PT, Chacko TK, Rovner AS, Goyal RK. Differences in contractile protein content and isoforms in phasic and tonic smooth muscles. Am J Physiol 1998;275(3 Pt 1):C684-92.

35. Somlyo AV, Khromov AS, Webb MR, et al. Smooth muscle myosin: regulation and properties. Philos Trans R Soc Lond B Biol Sci 2004;359:1921-30.

36. Injeti ER, Sandoval RJ, Williams JM, Smolensky AV, Ford LE, Pearce WJ. Maximal stimulation-induced in situ myosin light chain kinase activity is upregulated in fetal compared with adult ovine carotid arteries. Am J Physiol Heart Circ Physiol 2008;295:H2289-98.

37. Ihara E, Moffat L, Borman MA, Amon JE, Walsh MP, MacDonald JA. $\mathrm{Ca} 2+$-independent contraction of longitudinal ileal smooth muscle is potentiated by a zipper-interacting protein kinase pseudosubstrate peptide. Am J Physiol Gastrointest Liver Physiol 2009;297:G361-70.

38. Arner A, Pfitzer G. Regulation of cross-bridge cycling by $\mathrm{Ca} 2+$ in smooth muscle. Rev Physiol Biochem Pharmacol 1999;134:63-146.

39. Dean GE, Cargill RS 3rd, Macarak E, Snyder HM, Duckett JW, Levin R. Active and passive compliance of the fetal bovine bladder. J Urol 1997;158(3 Pt 2):1094-9.

40. Campbell S, Wladimiroff JW, Dewhurst CJ. The antenatal measurement of fetal urine production. J Obstet Gynaecol Br Commonw 1973;80:680-6. 